\title{
Long-period geomagnetic pulsations caused by the solar wind negative pressure impulse on 22 March 1979 (CDAW-6)
}

\author{
V. A. Parkhomov ${ }^{1}$, V. V. Mishin ${ }^{2}$ and L. V. Borovik ${ }^{1}$ \\ ${ }^{1}$ Irkutsk State Economy Academy, Lenin street 11, Irkutsk 3, 664003, Russia \\ ${ }^{2}$ Institute of Solar-Terrestrial Physics, Irkutsk 33, PO Box 4026, 664033, Russia
}

Received: 10 December 1996 / Revised: 30 June 1997 / Accepted: 21 July 1997

\begin{abstract}
An analysis is made of the long-period geomagnetic pulsations as recorded at seven Norilsk meridian stations $\left(\lambda=162^{\circ}\right.$, latitudinal range: $61^{\circ}$ $71^{\circ} \mathrm{N}$ ) following abrupt magnetospheric expansion during the storm of 22 March 1979 caused by a rapid decrease in solar wind density. As with the time interval following an abrupt contraction at the time of sudden storm commencement, there exist two types of pulsations in the pulsation spectra: latitude-independent $(T>400 \mathrm{~s})$ and latitude-dependent $(T<200 \mathrm{~s})$ pulsations. The first pulsation type is interpreted in terms of forced pulsations associated with magnetopause oscillations. The oscillation period is determined by plasma density in the boundary layer and by the radius of the magnetosphere $\left(T \sim \rho^{1 / 2} R^{4}\right)$. The latitudinal dependence of the period, amplitude and polarization of the second-type pulsations is in agreement with the resonance mechanism of their origin.
\end{abstract}

Keywords Geomagnetic pulsations · Solar wind · Magnetopause oscillations

\section{Introduction}

Abrupt changes in solar wind dynamic pressure during sudden storm commencements (SSC) and sudden impulses ( $\mathrm{Si}$ ) lead to the excitation of a broad spectrum of decaying geomagnetic pulsations (Psc) (Saito and Matsushita, 1967). Their frequency is a maximum at low latitudes and decreases with increasing latitude, which corresponds to the resonance theory of eigenoscillations of closed field lines in the magnetosphere (Southwood, 1974; Chen and Hasegawa, 1974). Psc pulsations have been the subject for both thoeretical (see

Correspondence to: V. V. Mishin e.g. Tamao, 1964; Southwood and Kivelson, 1990; Yumoto et al., 1990) and experimental study (see Nishida, 1978; Wilken et al., 1986; Parkhomov et al., 1985; Farrugia et al., 1989; Warnecke et al., 1990; Korotova and Sibeck, 1994). A number of regularities have been established and explained; however, the question of identifying the global magnetosonic mode still is a widely debated topic. This mode, corresponding to the Pc5 range, must be excited on the external $L$-shell of the magnetosphere with equal probability by both positive and negative pressure impulses of the solar wind (Southwood and Kivelson, 1990).

Since the wavelength of the global mode is about the size of the magnetosphere, at ground-based observatories it must be recorded over a wide latitude range with a constant period. To identify with confidence these oscillations requires: (1) simultaneous observations by a series of satellites in the outer magnetosphere and at an extended meridional chain of stations, and (2) studying time intervals not coincident with the active phase of a substorm which produces a number of disturbances, distorts the eigen-oscillations and makes their identification difficult. These requirements were not fully met in Knott et al. (1985) and Warnecke et al. (1990) which probably did not permit the global oscillations to be observed unambiguously.

In order to satisfy these conditions, we chose the storm of 22 March 1979. This storm was studied within the CDAW-6 program, and results were reported in a special issue of $J$. Geophys. Res., 90, V. A2, 1985. The emphasis was on the study of $\mathrm{SSC}^{*}$ and phenomena associated with the substorm that commenced at 1054 UT.

Latitude-independent 8-min pulsations were detected during the $\mathrm{SSC}^{*}$ of 22.03.1979 (Zolotukhina and Parkhomov, 1992) in data from the Norilsk meridian stations (see Table 1). The SSC ${ }^{*}$ was caused by a positive pressure impulse of the solar wind. Our study considers the regime of generation of Psc5 by a negative pressure impulse of 22.03.1979, as well as discussing the excitation mechanism for global oscillations of the magnetosphere. 
Table 1. $L$-value, geomagnetic latitude $(\Phi)$ and longitude $(\Lambda)$ of Norilsk meridian stations used

\begin{tabular}{lllll}
\hline $\mathrm{N}$ & Station & $\mathrm{L}$ & $\Phi^{\circ} \mathrm{N}$ & $\Lambda^{\circ} \mathrm{E}$ \\
\hline 1 & Isachenko & 9.8 & 71.01 & 162.8 \\
2 & Sterlegova & 8.1 & 69.5 & 162.0 \\
3 & U-Tareya & 6.9 & 67.6 & 162.9 \\
4 & Cresty & 5.9 & 65.6 & 162.2 \\
5 & Norilsk & 5.3 & 64.2 & 160.4 \\
6 & Turukhansk & 4.3 & 61.1 & 159.2 \\
\hline
\end{tabular}

\section{Observations and methods of analysis}

It is essential to recall the sequence of phenomena caused by the propagation of an interplanetary shock wave. At 0826 UT, as a result of an abrupt increase in dynamic and magnetic pressures within the shock front, the magnetopause became displaced from the geocentric distance $R=10 \mathrm{R}_{\mathrm{E}}$ to $R \sim 7 \mathrm{R}_{\mathrm{E}}$ (Wilken et al., 1986). At 1009:30 UT there was an abrupt decrease in solar wind density and pressure (by a factor of $\sim 3$ ), which led to a displacement of the magnetopause from the Earth from

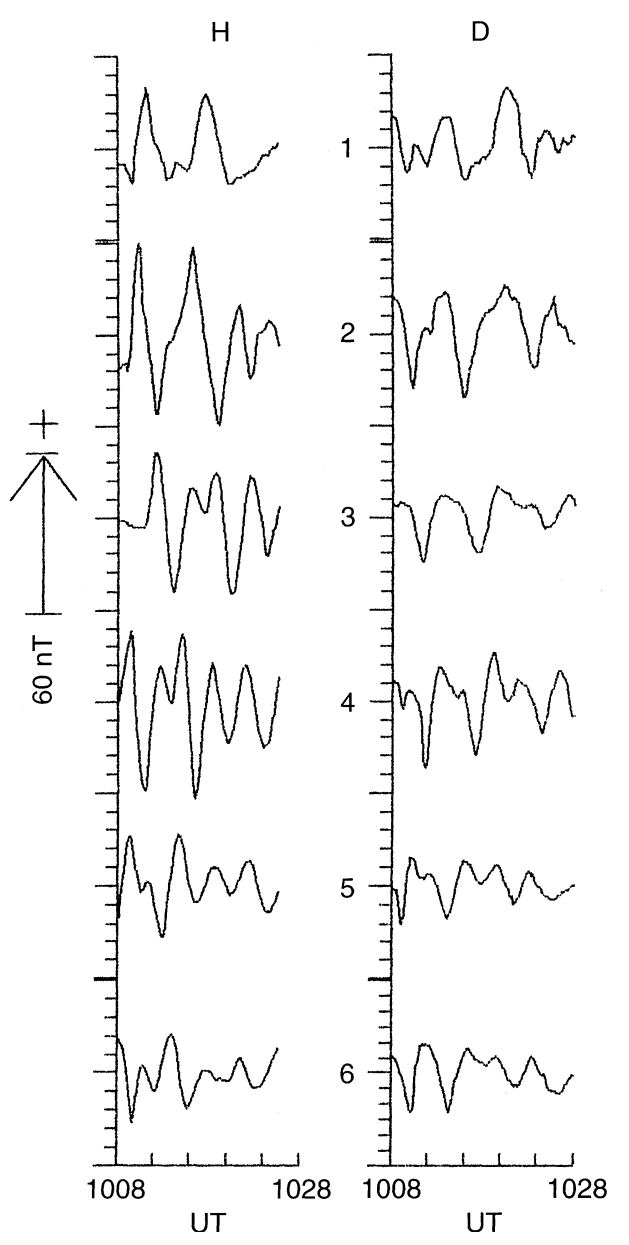

Fig. 1. Portions of magnetograms (the $H$ - and $D$-components) obtained from a chain of stations during a negative solar wind pressure impulse. 1, Is. Isachenko $(L=9.8) ; 2$, Cape Sterlegova $(L=8.1) ; 3$, Ust-Tareya $(L=6.9) ; 4$, Kresty $(L=5.9) ; 5$, Norilsk $(L=5.3) ; 6$, Turukhansk $(L=4.3)$
$R \sim 7 \mathrm{R}_{\mathrm{E}}$ to $R \sim 8.5 \mathrm{R}_{\mathrm{E}}$ (see Fig. 2 in Wilken et al., 1986). This displacement gave rise to the generation of oscillations recorded at the entire Norilsk meridian chain of stations. The oscillations continued till 1035 UT, decaying to the background of a positive bay disturbance, the beginning of which was determined to be at $1020 \mathrm{UT}$.

The initial analog recordings of the $H$ - and $D$ components, with the trend eliminated, are presented in Fig. 1 (Bobrov's magnetograph, time-base $40 \mathrm{~mm} / \mathrm{h}$ ). From these recordings, energy spectra were calculated. To determine polarization characteristics of the oscillations, the initial traces, after removing the trend, were subjected to band-pass filtering by a recursive sine band-pass filter in two frequency bands: $0-3 \mathrm{mHz}$ and 3-5 mHz.

\section{Geomagnetic pulsations}

Visual examination of the analog recordings of the $\mathrm{H}$ and $D$-components in Fig. 1 shows that oscillations at all stations start with a positive phase in the $H$-component and a negative phase in the $D$-component. The positive displacement in the $H$-component attracts particular attention, as there is a common view that a negative impulse is generated when the magnetosphere is expanding (Nishida, 1978). In our observations, the negative impulse, as such, can be identified only for stations where $L<4.3$. This can be illustrated by magnetogramms from middle and low-latitude stations (Fig. 2).

Oscillations at all stations of the chain were recorded over $25 \mathrm{~min}$, which corresponds to duration of the oscillations recorded by the geostationary satellite GEOS-2 (Knott et al., 1985).

In Fig. 1, a difference can be seen in the character of the oscillatory process and the evolution of the oscillations in the components. While in the $D$-component all stations record oscillations of the same type, with an apparent period $T \sim 400 \mathrm{~s}$, in the $H$-component the superposition of two main periods $(T \sim 400 \mathrm{~s}$ and $T \sim 240 \mathrm{~s})$ can be identified. Of special interest, we believe, is the difference in oscillation regimes at stations located at $L=8.1$ and $L=6.9$. At station 3, sinusoidal oscillations are recorded with a period of $T=240 \mathrm{~s}$, the maximum amplitude is clearly seen further south of this, e.g. at $L=5.9$. The period and form of the oscillations is in good agreement with electric field oscillations $\left(E_{\mathrm{D}}\right.$ component) observed by GEOS-2 (Knott et al., 1985).

At stations 1 and 2, trains of decaying oscillations with $T \sim 400 \mathrm{~s}$ are recorded, their amplitudes decreasing both to the north and to the south of the observation site.

\section{Analysis of energy spectra}

Energy spectra of the $H$ - and $D$-components of the geomagnetic pulsations are presented in Fig. 3. In the spectra of the $D$-component a spectral band can be identified, whose spectral density peaks at the frequency $f=2.3 \mathrm{mHz}(T=430 \mathrm{~s})$. This frequency with small 

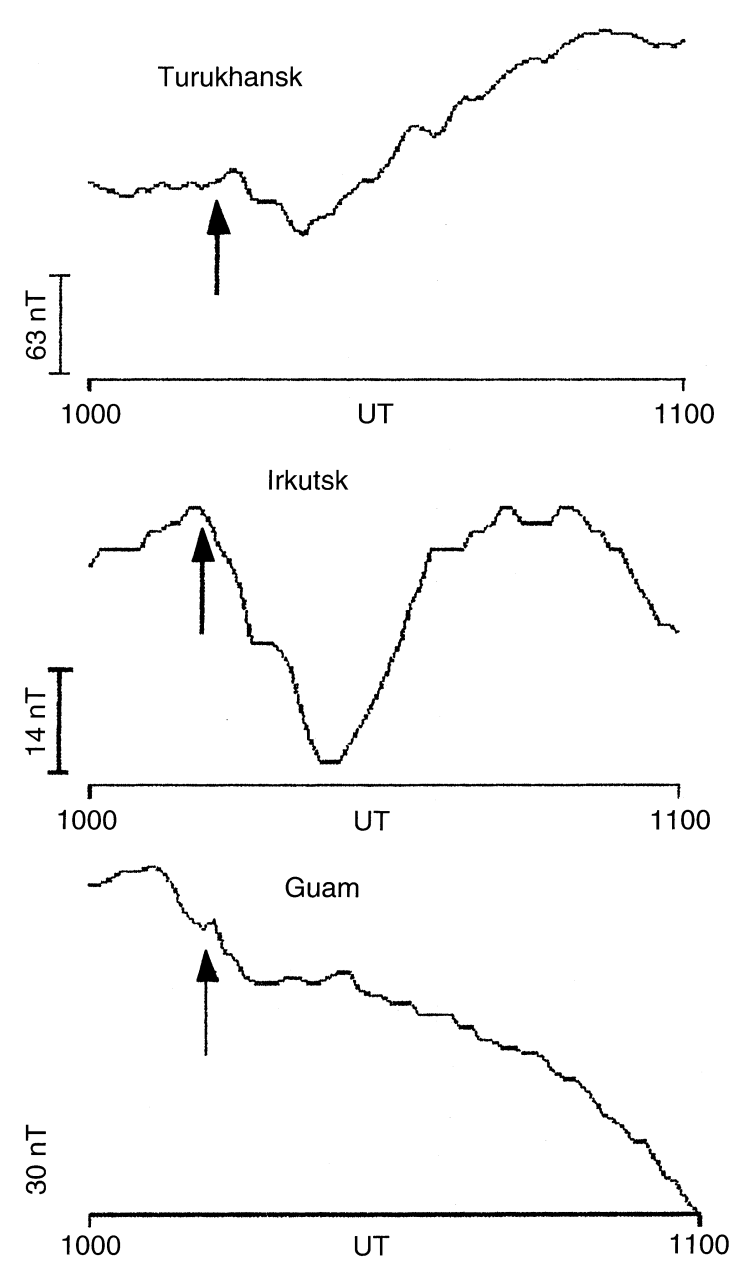

Fig. 2. Portions of magnetograms, the $H$-component from stations Turukhansk, Irkutsk $(L=2.2)$, Guam $(L=1)$, illustrating the negative impulse at 1009:30 arrow

variations is identified over the entire chain. The spectral density maximum at this frequency is also recorded at high-latitude stations. In general the pulsation energy decreases from north to south (by a factor of 2 over the entire chain).

Pulsations with $f=4-6 \mathrm{mHz}$ have a (typical for a resonance) dependence of frequency on latitude. The spectral density maximum at station 4 corresponds to a frequencey of $3.8 \mathrm{mHZ}(\mathrm{T}=265 \mathrm{~s})$ and $4.3 \mathrm{mHZ}$ $(T=230 \mathrm{~s})$ at station 6 . We must also point out the existence of the third and fourth harmonics in spectra from stations 4 and 5 .

More complicated regularities are traceable in spectra of the $H$-components. We are inclined to divide the spectra into two groups. The frequency of the main spectral peak $f=2.2 \mathrm{mHz}(450 \mathrm{~s})$ at the northernmost station differs little from the frequency $f=2.5 \mathrm{mHz}$ (400 s) which is traceable at the remaining stations in the chain. The pulsation energy maximum in this frequency band lies near $L=9.8$ and $L=8.1$.

The spectra from stations 4,5 and 6 show a reasonably good agreement: harmonics as high as the fourth harmonic show up in the spectra. The second spectral maximum corresponds to frequency of $3.8 \mathrm{mHz}$
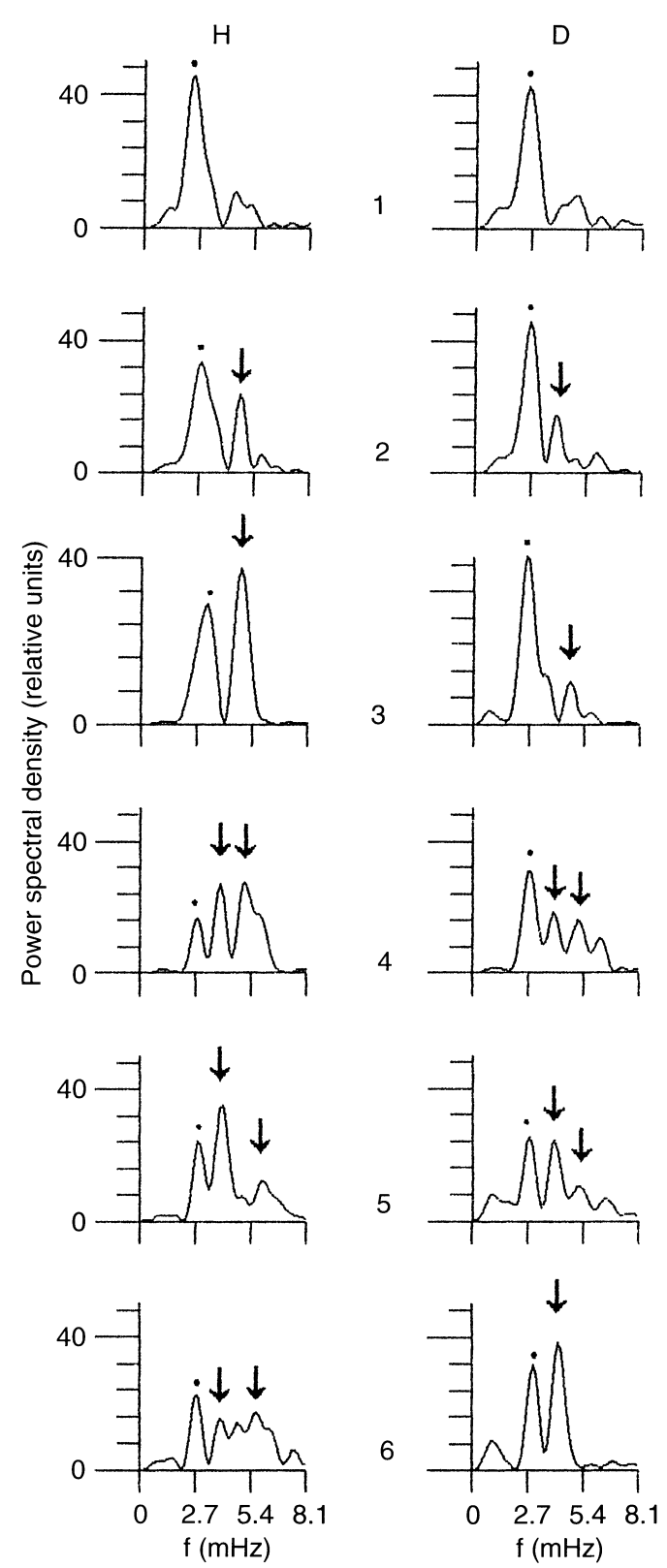

Fig. 3. Spectra of the recordings from Fig. 1: heavy dot, main spectral maximum; arrow, like spectral density peaks

(260 s). The frequency of the spectral maximum shows a broad trend from $4.5 \mathrm{mHz}(230 \mathrm{~s})$ at station 2 to $5.45 \mathrm{mHz}(T=180 \mathrm{~s})$ at station 6.

\section{Polarization of pulsations}

To analyze the polarization of the pulsations, the initial samples were filtered out using two pass-band filters with boundary frequencies $0-3 \mathrm{mHz}$ and $3-5.4 \mathrm{mHz}$, and the filtered-out sets were used to construct locus diagrams of the pulsations (Fig. 4). The pulsations, whose main spectral peak has a frequency of $2.3 \mathrm{mHz}$, are mainly left-handedly elliptically polarized (Fig. 4a), but the most intensive pulsations (at station 2) are polarized in the opposite direction (right-handed). 

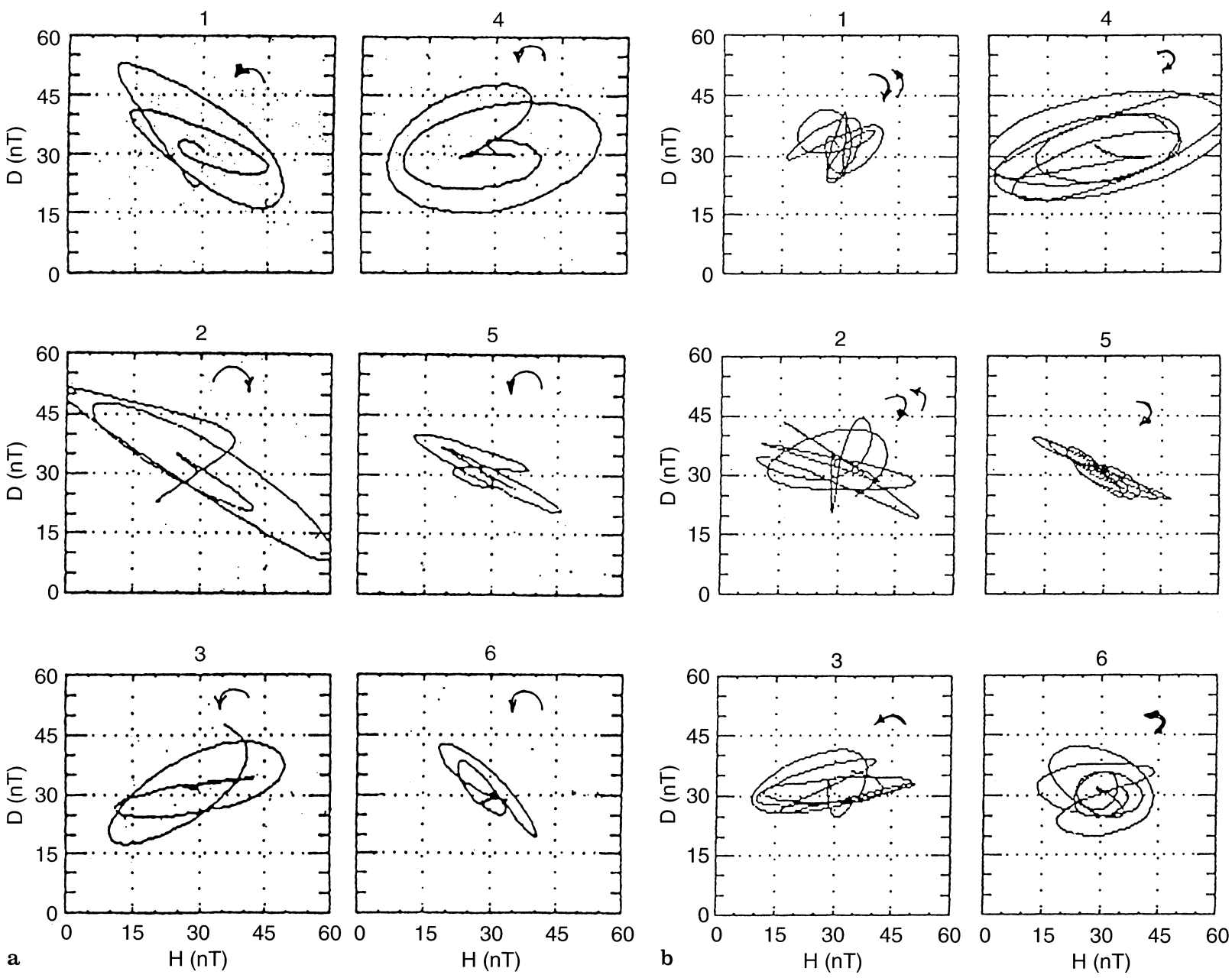

Fig. 4 a,b. Loci of oscillations ranging from: a, $0-3 \mathrm{mHz}$; b $3-5.4 \mathrm{mHz}$

Pulsations in the frequency range $3-5.4 \mathrm{mHz}$ are also elliptically polarized (Fig. 4b). At high-latitude stations the rotation direction of the locus diagram is unstable, it changes during the observation process. At $L<6.6$ such a direction is stable. The direction of the principal axis of the ellipse of polarization changes twice as one moves along the meridian: pulsations are polarized left-handedly at stations 3 and 6 and right-handedly at stations 4 and 5 .

\section{Discussion}

It is evident from the observations and analysis that in this event of an abrupt decrease in solar wind density and where, as a consequence, pressure decreases, the excitation of two types of pulsations occurs. These differ in morphological signatures.

The first type of pulsation is most clearly recorded at stations close to field lines mapping from the magnetopause to the ground, with a frequency $f=2.3 \mathrm{mHz}(T=430 \mathrm{~s})$. With small variations that may result from errors of measurement and processing, the $400 \mathrm{~s}$ pulsations are recorded at all stations in the chain, including the mid-latitude station Irkutsk for the north-south component. A maximum pulsation amplitude is recorded at station C. Sterlegova $(A=78 \mathrm{nT})$.

The second type of pulsation is most clearly recorded at stations U-Tareya and to the south. The dominant frequency of the pulsations at these stations with a maximum amplitude $(A=80 \mathrm{nT})$ is $f=3 \mathrm{mHz}$. These pulsations are characterized by a latitudinal dependence of frequency.

It is reasonable to suppose that the first type of pulsation is associated with magnetopause oscillations, and the second type is caused by intramagnetospheric resonances. We now try to explain this point of view. It follows from existing theoretical models by Southwood and Kivelson (1990), and Yumoto et al. (1990) that on the meridional profile the maximum amplitude of the excited standing waves (primarily, fast magnetosonic waves) must be on field lines as close to the magnetopause as possible and must decrease on both sides of it. The frequency of these oscillations, based on theoretical estimates and numerous observations, must be not lower than $3 \mathrm{mHz}$. In this case the excitation of Alfven resonators occurs at those $L$-shells where their frequency coincides with the fast magnetosound wave frequency arriving from the external source. What actually happens is that the fast magnetosound waves and Alfven 
waves are interrelated because of the inhomogeneity of the magnetosphere (Southwood and Saunders, 1985; Southwood and Kivelson, 1990). That the frequency of the low-frequency branch is invariable seems to be due to the fact that its value $(f=2.3 \mathrm{mHz})$ lies below the range of resonance oscillations. On the other hand, the range of oscillations of the second type falls within the resonance spectrum of the magnetosphere. Therefore, these oscillations exhibit typical properties of resonance oscillations: the presence of harmonics and the dependence of resonance frequency on latitude (Fig. 3). In Warnecke et al. (1990) it is shown that fast magnetosound waves on the ground produce large disturbances of the $D$-component of the geomagnetic field, while Alfven resonances give rise to disturbance in the $H$-component. Hence it is possible to explain these differences in the distribution of the $D$ - and $H$-components along the Norilsk meridian, namely the presence of two maxima in the $H$-component compared with a single maximum in the $D$-component. It is natural to associate the high-latitude maximum (in both components) with the magnetopause, and the additional maximum in the $H$-component can be attributed to the excitation of a resonance Alfven wave. The resonance origin of this additional maximum is also suggested by the reversal of the polarization of the second-type of oscillations near the plasmapause. The point here is that the reversal of polarization of the Alfven eigen-oscillations occurs where they undergo a resonance excitation due to a fast magnetosound wave (Southwood, 1974). The probability of such an excitation is higher near the plasmapause where the Alfven velocity (and also the wave frequency) change drastically over small radial distances. The presence of higher harmonics at stations located on closed field lines, is associated with the excitation of standing resonance oscillations at multiple (for a given latitude) harmonics. At stations located north of the magnetopause projection, it is natural that no such resonances are observed.

One issue remains open: what is the actual outer boundary of the magnetospheric resonator: the magnetopause, as is commonly assumed (Southwood and Kivelson, 1990; Yumoto et al., 1990) or the bow shock (Harrold and Samson, 1992)? We are inclined to conjecture that the fast magnetosound wave-guide boundary lies outside the magnetopause. As is evident from Fig. 1, the oscillation amplitude of the $D$-component at stations C. Sterlegova and Isachenko differ little, although the latter is at a much larger distance from the magnetopause $(L=8.5)$. The point here is that the fast magnetosound wave has a finite transmission coefficient through a sharp magnetopause already in its approximation by the hydromagnetic tangential discontinuity (without transition layer, McKenzie, 1970), and the presence of a blurred boundary layer must contribute to an increase of its transparency for the fast magnetosound wave.

We now discuss the question of the source of the observed pulsations. It is obvious that it is caused by a sharp negative impulse of solar wind density and the subsequent expansion of the magnetosphere. An ab- rupt displacement of the boundary must be accompanied by its oscillations near the equilibrium position (Mishin, 1993). The period of these time-decaying oscillations is several minutes and depends greatly on the magnetosphere's radius, as well as on the plasma density in the boundary layer: $T \sim \rho^{1 / 2} R^{4}$. Since this formula was obtained by Mishin (1993) in the spherical symmetry approximation and the real magnetosphere represents a paraboloid, this formula gives an underestimated value of the period. Therefore, instead of $R=R_{0}$ (radius of the subsolar point), it is necessary to take a "mean" radius of the dayside magnetosphere, $R=1.3 R_{0}$. The model by Mishin (1993) then gives, for $R_{0} \sim 8.5 \mathrm{R}_{\mathrm{E}}$, a value of the period close to the observed one $(T \sim 400 \mathrm{~s})$.

Almost the same value of the magnetopause oscillation period was also obtained theoretically by Freeman et al. (1995) in the Newton-Busemann approximation to the hypersonic solar wind - magnetosphere interaction. They showed that the $e$-folding time of the linear magnetopause oscillations is about $40 \%$ of its period, and in response to the small impulsive change in solar wind dynamic pressure $(10 \%)$ the magnetosphere exhibits an oscillatory compressional oscillation of about one cycle. A change in solar wind pressure was significantly stronger $(>100 \%)$ for the decompression impulse of our concern. Therefore, the magnetopause changes its shape due to the effect of the front of changed pressure (Southwood and Kivelson, 1990; Sibeck, 1990). The excitation time of the oscillations of the dayside boundary can be inferred from the time taken by the region of decreased pressure of the incident flux to be carried away from the near-noon stagnation region ahead of the magnetopause nose. Taking the longitudinal size of this region in local time to be $2 \mathrm{~h}$ (which gives a linear size of $\sim R / 2$ ) and the velocity in the stagnation region $v=50 \mathrm{~km} / \mathrm{s}$ we obtain $\tau \sim 10^{3} \mathrm{~s}$. This time is comparable with the time of recording the pulsations.

This mechanism is in agreement with conclusions drawn in Knott et al. (1985) about the excitation of poloidal oscillations at the magnetopause nose. The magnetopause decompression, determined on GEOS-2 and on the ground, was accompanied by Pc5 pulsations. The difference in the pulsation regime observed on the ground during the magnetopause compression and decompression (for a different event) is also mentioned by Farrugia et al., 1989. However, the cited reference does not analyze in detail the spectral and amplitude characteristics of the pulsations at the meridional chain for magnetospheric decompression.

Magnetopause oscillations are the external periodic source that drives oscillations of the magnetosphere. Since the period of magnetopause oscillations appears to be in excess of the period of resonances, noneigenoscillations are recorded on the ground during the lifetime of the boundary oscillations. Because their wavelength is large (for the global mode, the value of the wave number $k=1 / R)$, the amplitude of these oscillations decreases with the depth of the magnetosphere (and, accordingly, along the Norilsk meridian) relatively weakly and are therefore well seen on the ground. 
In addition to forced oscillations in the magnetosphere, resonance oscillations must reach the steady state following 1 period (Leonovich and Mazur, 1989) because the original rarefaction impulse is a broadbanded one and contains the overall spectrum of magnetospheric resonances.

Note that the mechanism considered here does not overthrow our earlier proposed excitation mechanism (Parkhomov et al., 1985) for the magnetospheric resonator across the cusp which can make a significant contribution in the near-noon region.

Acknowledgements. We are grateful to Mr. V. G. Mikhalkovsky for his assistance in preparing the English version of the manuscript. This work was partially supported by a Grant from the Russian Foundation for Fundamental Research (Grant 95-05-15471-a).

Topical Editor K.-H. Glaßmeier thanks C. J. Farrugia and B. Wilken for their help in evaluating this paper.

\section{References}

Chen, L., and A. Hasegawa, A theory of long period magnetic pulsations -1, J. Geophys. Res., 79, 1024, 1974.

Farrugia, C. J., M. P. Freeman, S. W. H. Cowley, D. J. Southwood, and M. Lockwood, Pressure driven magnetopause motions and attendant response on the ground, Planet. Space Sci., 37, 589607, 1989

Freeman, M. P., N. C. Freeman, and C. J. Farrugia, A linear perturbation analisis of magnetopause motion in the NewtonBusemann limit, Ann. Geophysicale, 13, 907-918, 1995.

Harrold, B. G., and J. C. Samson, Standing ULF modes of the magnetosphere: theory, Geophys. Res. Lett., 19, 1811-1814, 1992.

Knott, K., A. Pedersen, and U. Wedeken, GEOS 2 electric field observations during a sudden commencement and subsequent substorms, J. Geophys. Res., 90, 1283-1288, 1985.

Korotova G. I., and D. G. Sibeck, Generation of ULF magnetic pulsations in response to sudden variations in solar wind dynamic pressure, in Solar Wind Sources of Magnetospheric Ultra-Low-Frequency Waves, pp. 265-271, American Geophysical Union, 1994.

Leonovich, A. S., and V. A. Mazur, Resonance excitation of standing Alfven waves in an axisymmetric magnetosphere (nonstationary oscillations), Planet. Space Sci., 37, 1109-1116, 1989.

McKenzie J. F., Hydromagnetic wave interaction with the magnetopause and with the bow shock, Planet. Space Sci., 18, 1-23, 1970.

Mishin, V. V., Accelerated motions of the magnetopause as a trigger of the Kelvin-Helmholtz instability, J. Geophys. Res., 98, 21365-21371, 1993.

Nishida, A., Geomagnetic Diagnosis of the Magnetosphere. Springer-Verlag, New York Heidelberg Berlin, 1978.

Parkhomov, V. A., V. I. Lukovnikova, V. V. Stupin, and V. V. Mishin, Long-period Psc4-6 pulsations during SSC, Issled. Geomagn., Aeronom., Fiz. Solntsa, 74, 41-49, 1985 (in Russian).

Saito, T., and S. Matsushita, Geomagnetic pulsations associated with sudden commencements and sudden impulses, Planet. Space Sci., 15, 579-585, 1967.

Sibeck, D.G., A model for the transient magnetospheric response to sudden solar wind dynamic pressure variations, J. Geophys. Res., 95, 3755-3771, 1990.

Southwood, D. J., Some features of field line resonances in the magnetosphere, Planet. Space Sci., 22, 283, 1974.

Southwood, D. J., and M. A. Saunders, Curvature coupling of slow and Alfven MHD waves in a magnetotail field configuration, Planet. Space Sci., 33, 127-134, 1985.

Southwood, D. J., and M. Kivelson, The magnetohydrodynamic response of the magnetospheric cavity to changes in solar wind pressure, J. Geophys. Res., 95, 2301-2309, 1990.

Tamao, T., A hydromagnetic interpretation of geomagnetic SSC, Rep. Ionos. Space Res. Jpn., 18, 16, 1964.

Warnecke, J., H. Luhr, and K. Takahashi, Observational features of field line resonances excited by solar wind pressure variations on 4 September 1984, Planet. Space Sci., 12, 1517-1531, 1990.

Wilken, B., D. N. Baker., P. R. Higbie, T. A. Fritz, W. P. Olson, and K. A. Pfitzer, Magnetospheric configuration and energetic particle effects with SSC: a case study of the CDAW-6 event on 22.03.1979, J. Geophys. Res., 91, 1459-1473, 1986.

Yumoto, K., S. Watanabe, and H. Oya, MHD response of a model magnetosphere to magnetopause perturbations, in Proceedings of Research Institute of Atmospherics, Nagoya University, 37, 17-36, 1990.

Zolotukhina, N. A., and V. A. Parkhomov, The longitudinal assymetry of geomagnetic phenomena as deduced by considering the sudden substorm commencement of March 22, 1979 (CDAW-6), Issled. Geomagn., Aeronom., Fiz. Solntsa, 97, 55-66, 1992 (in Russian). 